\title{
Performance Evaluation of Wireless Sensor Networks for Mobile Sink Considering Consumed Energy Metric
}

\author{
Tao Yang*, Makoto Ikeda*, Gjergji Mino*, Leonard Barolli ${ }^{\dagger}$, Arjan Durresi ${ }^{\ddagger}$ and Fatos Xhafa ${ }^{\S}$ \\ * Graduate School of Engineering \\ Fukuoka Institute of Technology (FIT) \\ 3-30-1 Wajiro-Higashi, Higashi-Ku, Fukuoka 811-0295, Japan \\ Email: bd07003@bene.fit.ac.jp,makoto.ikd@acm.org,bd09002@bene.fit.ac.jp \\ ${ }^{\dagger}$ Department of Information and Communication Engineering \\ Fukuoka Institute of Technology (FIT) \\ 3-30-1 Wajiro-Higashi, Higashi-Ku, Fukuoka 811-0295, Japan \\ Email: barolli@fit.ac.jp \\ $\ddagger$ Department of Computer and Information Science \\ Indiana University Purdue University at Indianapolis (IUPUI) \\ 723 W. Michigan Street SL 280 Indianapolis, IN 46202, USA \\ durresi@cs.iupui.edu \\ $\S$ Technical University of Catalonia \\ Department of Languages and Informatics Systems \\ C/Jordi Girona 1-3, 08034 Barcelona, Spain \\ E-mail:fatos@lsi.upc.edu
}

\begin{abstract}
Sensor networks are a sensing, computing and communication infrastructure that are able to observe and respond to phenomena in the natural environment and in our physical and cyber infrastructure. The sensors themselves can range from small passive micro-sensors to larger scale, controllable weather-sensing platforms. To reduce the consumed energy of a large scale sensor network, we consider a mobile sink node in the observing area. In this work, we investigate how the sensor network performs in the case when the sink node moves. We compare the simulation results for two cases: when the sink node is mobile and stationary considering lattice and random topologies using AODV protocol. The simulation results have shown that for the case of mobile sink, the consumed energy is better than the stationary sink (about half of stationary sink in lattice topology). Also for mobile sink, the consumed energy of lattice topology is better than random topology.
\end{abstract}

Keywords-WSN; Radio Model; Consumed Energy; WSN Topology.

\section{INTRODUCTION}

In the Wireless Sensor Network (WSN), a large number of nodes, having both computing power and wireless communication capability, are embedded in the environment, collect sensor data, and report to the sink. WSN, have wide range of applications and can be categorized into monitoring space and monitoring things. WSN can be considered as a special type of Ad Hoc wireless sensor networks, where sensor nodes are, in general, stationary. A unique feature of sensor networks is the cooperative effort of sensor nodes. Sensor nodes are usually fitted with on-board processors. Instead of sending the raw data to the nodes responsible for the fusion, they use their processing abilities to locally carry out simple computations and transmit only the required and partially processed data. A sensor system normally consists of a set of sensor nodes operating on limited energy and a base system without any energy constraint called sink. Typically, the sink serves as the gathering point for the collected data. The sink also broadcasts various control commands to sensor nodes.

There are many applications of senor networks. For instance, in military application, the rapid deployment, selforganization, and fault-tolerance characteristics of sensor nodes make them a promising, surveillance, reconnaissance, and targeting systems. In health car, sensor nodes can be used to monitor patients and assist disabled patients. Other applications include managing inventory, monitoring product quality, and monitoring disaster areas.

Recently, there are many research work for sensor networks [1], [2], [3], [4]. In our previous work [5], we implemented a simulation system for sensor networks considering different protocols and different propagation radio models. In this paper, we study a particular application of WSN for event-detection and tracking. The application is based on the assumption that WSN present some degree of spatial redundancy. For instance, whenever an event happens, a certain event data is transmitted to the sink node. Because of the spatial redundancy, we can tolerate some packet loss, as long as the required detection or event-reliability holds. This reliability can be formulated as the minimum number 


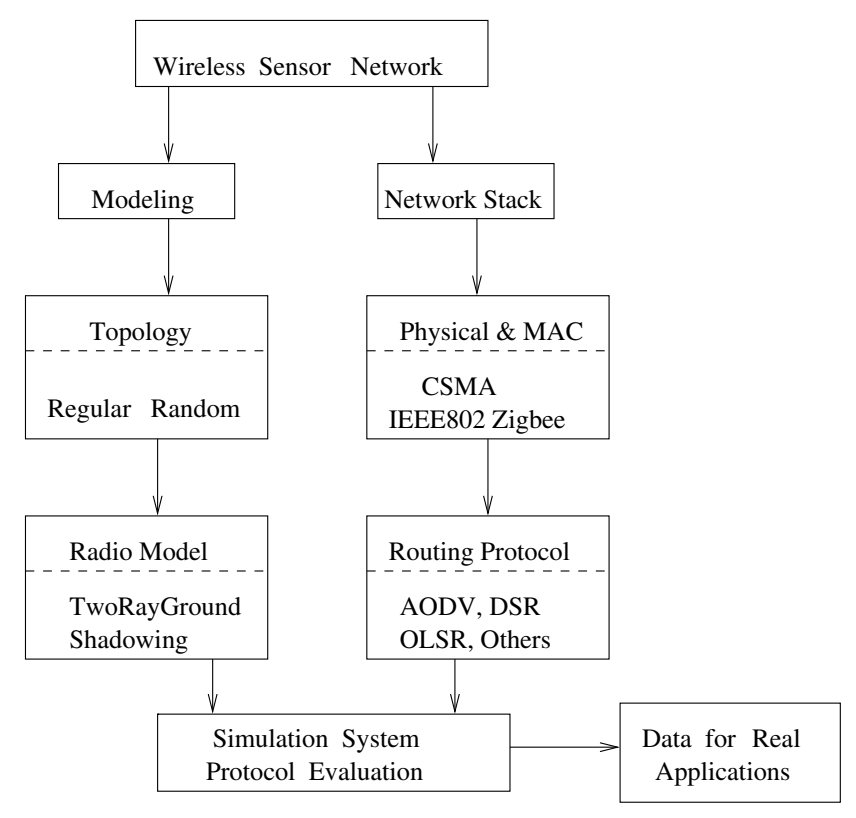

Figure 1. Network simulation model.

of packets required by the sink node in order to re-construct the event field. We want to investigate the performance of WSN for different topologies considering a single mobile sink. In the large scale network, the sink node is faraway from the sensor nodes. For this reason, it is needed more energy to send the sensed data. To reduce the consumed energy of sensor node we consider the mobile sink for the large scale networks.

The remainder of the paper is organized as follows. In Section II, we explain the proposed network simulation model. In Section III, we discuss the consumed energy. In Section IV, we show the simulation results. Conclusions of the paper are given in Section V.

\section{Proposed Network Simulation Model}

In our WSN, every node detects the physical phenomenon and sends back to the sink node the data packets. We suppose that the sink node is more powerful than sensor nodes. In our previous work, the sink node was stationary. In this work, we consider that the sink is mobile. We analyze the performance of the network in a fixed time interval. This is the available time for the detection of the phenomenon and its value is application dependent.

Proposed network simulation model is shown in Fig. 1. In our work, for simulation system implementation, we considered modeling and network stack. In this paper, we consider that a mobile sink is moving randomly in the WSN field. In Fig. 2 is shown one pattern of mobile sink path. We evaluated the consumed energy of AODV protocol

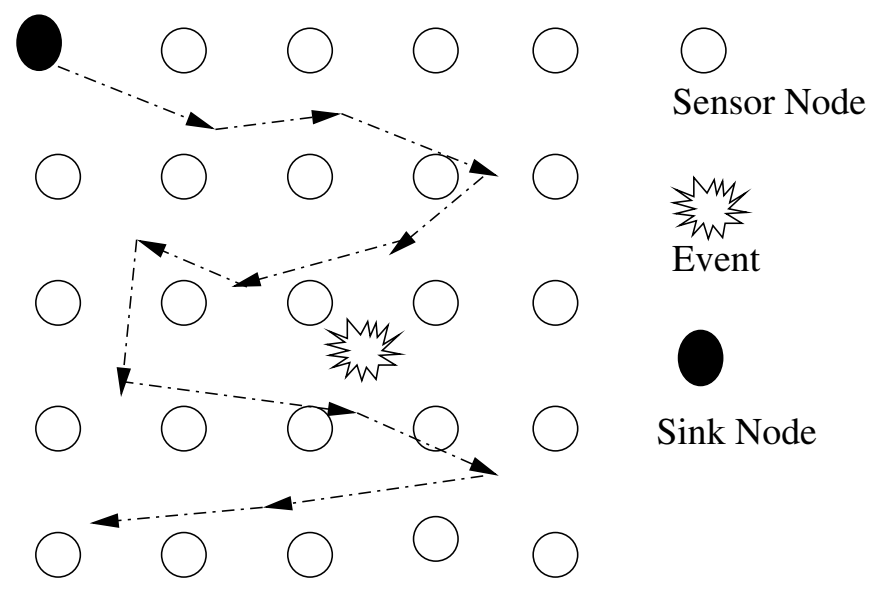

Figure 2. One pattern of mobile sink path.

using TwoRayGround radio model for the lattice and random topologies.

\section{A. Topology}

For the physical layout of the WSN, two types of topologies has been studied so far: random and lattice topologies. In the former, nodes are supposed to be uniformly distributed, while in the latter one nodes are vertexes of particular geometric shape, e.g. a square grid. For lattice topology, in order to guarantee the connectedness of the network we should set the transmission range of every node to the step size, $d$, which is the minimum distance between two rows (or columns) of the grid. In fact, by this way the number of links that every node can establish (the node degree D) is 4 . Nodes at the borders have $D=2$.

In the case of random networks, we suppose that the coordinates in the Euclidean plane of every sensor are random variables uniformly distributed in the interval $[0, L] \times[0, L]$. Snapshots of lattice and random networks generated in simulations are shown in Fig. 3 and Fig. 4, respectively.

\section{B. Radio Model}

In order to simulate the detection of a natural event, we used the libraries from Naval Research Laboratory (NRL) [7]. In this framework, a phenomenon is modeled as a wireless mobile node. The phenomenon node broadcasts packets with a tunable synchrony or pulse rate, which represents the period of occurrence of a generic event ${ }^{1}$. These libraries provide the sensor node with an alarm variable. The alarm variable is a timer variable. It turns off the sensor if no event is sensed within an alarm interval. In addition to the

\footnotetext{
${ }^{1}$ As a consequence, this model is for discrete events. By setting a suitable value for the pulse rate, it is possible in turn to simulate the continuous signal detection such as temperature or pressure.
} 


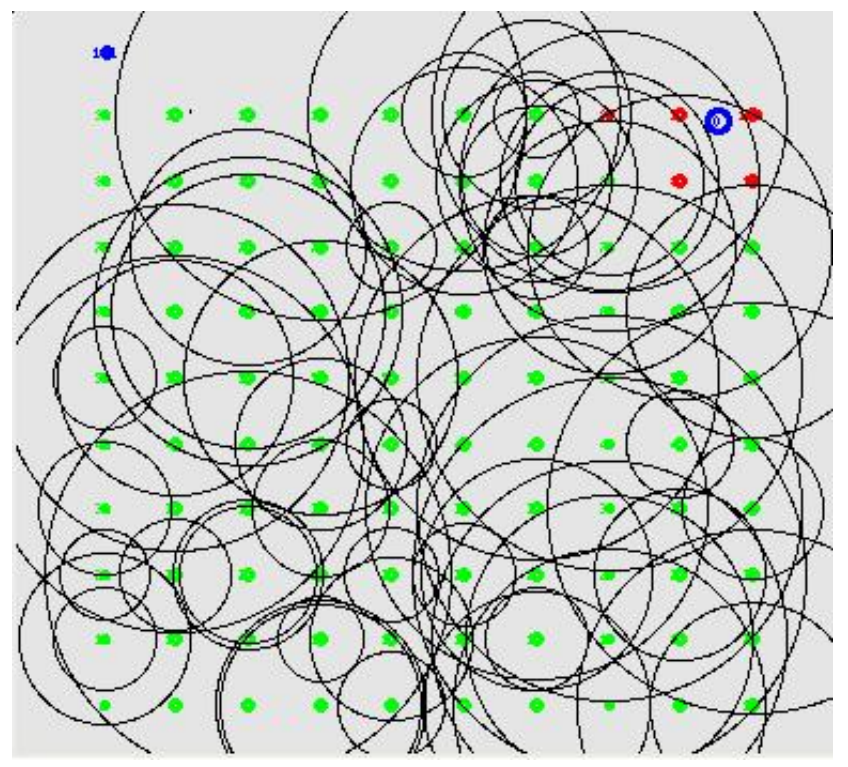

Figure 3. An example of lattice network.

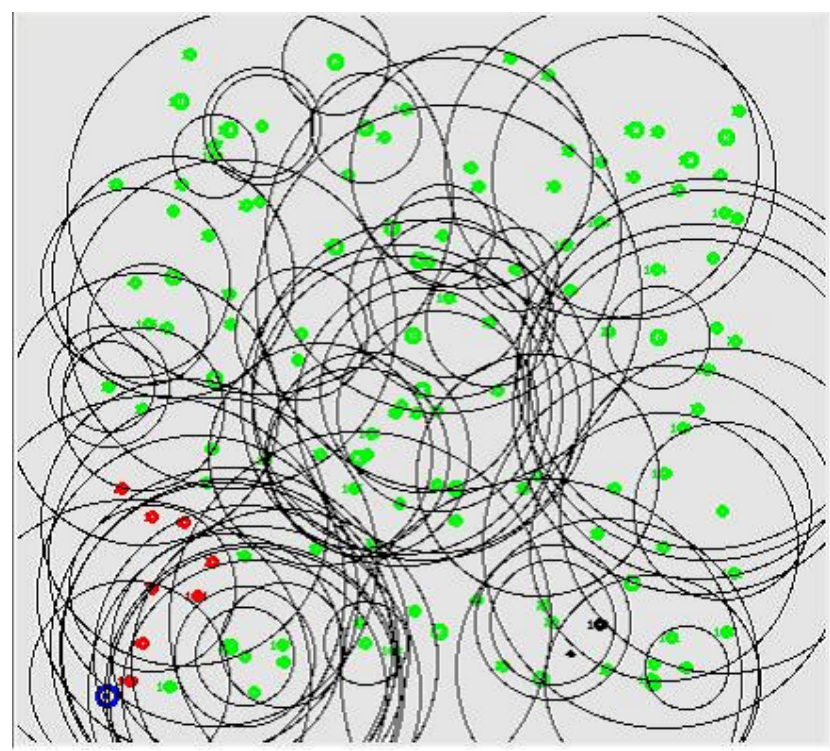

Figure 4. An example of random network.

sensing capabilities, every sensor can establish a multi-hop communication towards the sink by means of a particular routing protocol.

We assume that the MAC protocol is the IEEE 802.11 standard. This serves to us as a baseline of comparison for other contention resolution protocols. The receiver of every sensor node is supposed to receive correctly data bits if the received power exceeds the receiver threshold, $\gamma$. This threshold depends on the hardware ${ }^{2}$. As reference, we select parameters values according to the features of a commercial device (MICA2 OEM). In particular, for this device, we found that for a carrier frequency of $f=916 \mathrm{MHz}$ and a data rate of $34 \mathrm{KBaud}$, we have a threshold (or receiver sensitivity) $\left.\gamma\right|_{d B}=-118 \mathrm{dBm}$ [8]. The calculation of the phenomenon range is not yet optimized and the phenomenon propagation is assumed to follow the propagation laws of the radio signals. In particular, the emitted power of the phenomenon is calculated according to a TwoRayGround propagation model. [9]. The received power $P_{r}$ at a certain distance $d$ is the same along all directions in the plane ${ }^{3}$. For example, in the case of Line Of Sight (LOS) propagation of the signal, the Friis formula predicts the received power as:

$$
\begin{aligned}
& P_{r}(d)=P_{t}-\beta(\mathrm{dB}), \\
& \beta=10 \log \left(\frac{(4 \pi d)^{2} L}{G_{t} G_{r} \lambda^{2}}\right)
\end{aligned}
$$

where $G_{r}$ and $G_{t}$ are the antenna gains of the receiver and the transmitter, respectively, $\lambda$ is the wavelength of the signal, $L$ the insertion loss caused by feeding circuitry of the antenna, and $\beta$ is the propagation pathloss. For omni-antennas, $G_{R}=G_{t}=1$. The signal decay is then proportional to $d^{2}$. A more accurate model is Two-RayGround model, where in addition to the direct ray from the transmitter towards the receiver node, a ground reflected signal is supposed to be present. Accordingly, the received power depends also on the antenna heights and the pathloss is:

$$
\beta=10 \log \left(\frac{(4 \pi d)^{4} L}{G_{t} G_{r} h_{t} h_{r} \lambda^{2}}\right)
$$

where $h_{r}$ and $h_{t}$ are the receiver and transmitter antenna heights, respectively. The power decreases faster than Eq. (1). The formula in Eq. (2) is valid for distances $d>d_{c}\left(d_{c}\right.$ is the distance threshold of signal LOS propagation), that is far from the transmitting node.

\section{Energy Model}

The energy model concerns the dynamics of energy consumption of the sensor. A widely used model is as follows [10]. When the sensor transmits $k$ bits, the radio circuitry consumes an energy of $k P_{T x} T_{B}$, where $P_{T x}$ is the power required to transmit a bit which lasts $T_{B}$ seconds. By adding the radiated power $P_{t}(d)$, we have:

$$
E_{T x}(k, d)=k T_{B}\left(P_{T x}+P_{t}(d)\right) .
$$

\footnotetext{
${ }^{2}$ Other MAC factors affect the reception process, for example the Carrier Sensing Threshold (CST) and Capture Threshold (CT) of IEEE.802.11 used in NS-2.

${ }^{3} \mathrm{We}$ are considering $2 \mathrm{D}$ networks, but similar results hold also in the more general case of tridimensional networks.
} 
Since packet reception consumes energy, by following the same reasoning, we have:

$$
E(k, d)=k P_{T x} T_{B}+k T_{B} P_{t}(d)+k P_{R x} T_{B}
$$

where $P_{R x}$ is the power required to correctly receive (demodulate and decode) one bit.

\section{Interference}

In general, in every wireless network the electromagnetic interference of neighboring nodes is always present. The interference power decreases the Signal-to-Noise-Ratio (SNR) at the intended receiver, which will perceive a lower bit and/or packet error probability. Given a particular node, the interference power depends on how many transmitters are transmitting at the same time of the transmission of the given node. In a WSN, since the number of concurrent transmissions is low because of the low duty-cycle of sensors, we can neglect the interference. In other words, if we define duty-cycle as the fraction between the total time of all transmissions of sensor data and the total operational time of the network, we get always a value less than 0.5 . In fact, the load of each sensor is $\ll 1$ because sensors transmit data only when an event is detected [10]. However, it is intuitive that in a more realistic scenario, where many phenomena trigger many events, the traffic load can be higher, and then the interference will worsen the performance.

\section{E. Routing Protocols}

In this work, we consider AODV protocol. The AODV is an improvement of DSDV to on-demand scheme. It minimize the broadcast packet by creating route only when needed. Every node in network maintains the route information table and participate in routing table exchange. When source node wants to send data to the destination node, it first initiates route discovery process. In this process, source node broadcasts Route Request (RREQ) packet to its neighbors. Neighbor nodes which receive RREQ forward the packet to its neighbor nodes. This process continues until RREQ reach to the destination or the node who know the path to destination. When the intermediate nodes receive RREQ, they record in their tables the address of neighbors, thereby establishing a reverse path. When the node which knows the path to destination or destination node itself receive RREQ, it send back Route Reply (RREP) packet to source node. This RREP packet is transmitted by using reverse path. When the source node receives RREP packet, it can know the path to destination node and it stores the discovered path information in its route table. This is the end of route discovery process. Then, AODV performs route maintenance process. In route maintenance process, each node periodically transmits a Hello message to detect link breakage.

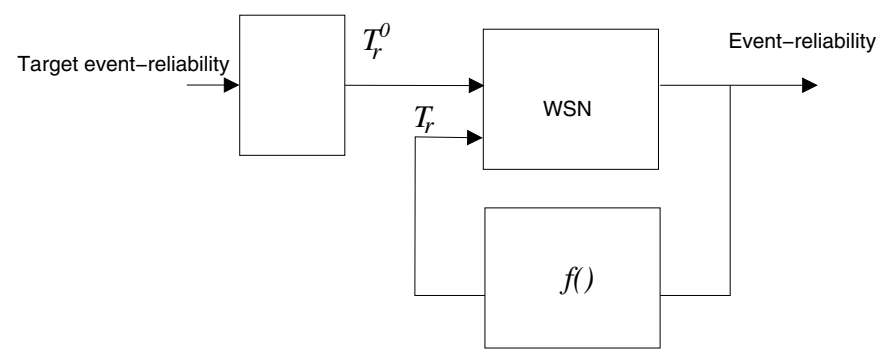

Figure 5. Representation of the transport based on the event-reliability.

\section{F. Event Detection and Transport}

For event detection and transport, we use the data-centric model similar to [11], where the end-to-end reliability is transformed into a bounded signal distortion concept. In this model, after sensing an event, every sensor node sends sensed data towards the sink. The transport used is a UDPlike transport. While this approach reduces the complexity of the transport protocol and well fit the energy and computational constraints of sensor nodes, the event-reliability can be guaranteed to some extent because of the spatial redundancy. The sensor node transmits data packets reporting the details of the detected event at a certain transmission rate ${ }^{4}$. The setting of this parameter, $T_{r}$, depends on several factors, as the quantization step of sensors, the type of phenomenon, and the desired level of distortion perceived at the sink. In [12], the authors used this $T_{r}$ as a control parameter of the overall system. For example, if we refer to event-reliability as the minimum number of packets required at sink in order to reliably detect the event, then whenever the sink receives a number of packets less than the event-reliability, it can instruct sensor nodes to use a higher $T_{r}$. This instruction is piggy-backed in dedicated packets from the sink. This system can be considered as a control system, as shown in Fig. 5, with the target event-reliability as input variable and the actual event-reliability as output parameter. The target event-reliability is transformed into an initial $T_{r}^{0}$. The control loop has the output event-reliability as input, and on the basis of a particular non-linear function $f(\cdot), T_{r}$ is accordingly changed. We do not implement the entire control system, but only a simplified version of it. For instance, we vary $T_{r}$ and observe the behavior of the system in terms of the mean number of received packets. In other words, we open the control loop and analyze the forward chain only.

\section{CONSUMED ENERGY}

As long as the WSN is being used, a certain amount of energy will be consumed. The energy consumption rate directly affects the life-time of the network, i.e. the time after which the WSN is unusable. The energy depletion is a function of the reporting rate as well as the density of the

\footnotetext{
${ }^{4}$ Note that in the case of discrete event, this scheme is a simple packet repetition scheme.
} 
Table I

TOPOLOGY SETTINGS

\begin{tabular}{|c|c|}
\hline \multicolumn{2}{|c|}{ Lattice } \\
\hline \hline Step & $d=\frac{L}{\sqrt{N}-1} \mathrm{~m}$ \\
\hline Service Area Size & $L^{2}=(800 \times 800) \mathrm{m}^{2}$ \\
\hline Number of Nodes & $N=64,100,256$ \\
\hline Transmission Range & $r_{0}=d$ \\
\hline \hline \multicolumn{2}{|c|}{ Random } \\
\hline Density(nodes/m $\left.{ }^{2}\right)$ & $\rho \in\left\{25 \cdot 10^{-6}, 2 \cdot 10^{-4}\right\}$ \\
\hline Transmission Range $(\mathrm{m})$ & $r_{0}=180$ \\
\hline
\end{tabular}

sensor network. Recall that the density of the network in the event-driven scenario correlates with the number of nodes that report their data. Accordingly, we define the consumed energy by the network in the detection interval $\tau$ as:

$$
\bar{\Delta}(\tau)=\frac{N E_{I}-\sum_{i=1}^{N} e_{i}(\tau)}{N \tau}
$$

where $e_{i}(t)$ is the node energy at time $t$ and the means are computed over the number of nodes. The number of nodes $N$ is set as power of integers in order to analyse the behaviour of the scaled versions of the network.

\section{Simulation Results}

In this section, we present the simulation results of our proposed WSN. We simulated the network by means of NS-2 simulator, with the support of NRL libraries ${ }^{5}$. In this work, we simulated two patterns considering stationary sink and mobile sink. For AODV routing protocol, the sample averages of Eq. (4) are computed over 20 simulation runs, and they are plotted from Fig. 6 to Fig. 8. The results for lattice topology are shown in Fig. 6 and Fig. 7.

In Tables I and II, we summarise the values of parameters used in our WSN. Let us note that the power values concern the power required to transmit and receive one bit, respectively. They do not refer to the radiated power at all. This is also the energy model implemented in the widely used NS-2 simulator.

The consumed energy in case of mobile sink is about half of the case of stationary sink as shown in Fig. 6 and Fig. 7. The results of random topology are plotted in Fig. 8. The depletion of random topology is better than lattice topology when the number of nodes are less than 64 . When the number of nodes is large than 64 , the Depletion is worse than random topology. The explanation of this effect is not simple, because it is intermingled with the dynamics of MAC and routing protocols. However, intuitively we can say that the on-demand routing protocols are affected by the presence of the mobile sink. It is worth noting that AODV and other protocols cannot use unidirectional links. On the other hand,

\footnotetext{
${ }^{5}$ Since the number of scheduler events within a simulated WSN can be very high, we applied a patch against the scheduler module of NS-2 in order to speed up the simulation time [13].
}

Table II

RADIO MODEL AND SYSTEM PARAMETERS.

\begin{tabular}{|c|c|}
\hline \multicolumn{2}{|c|}{ Radio Model Parameters } \\
\hline Path Loss Coefficient & $\alpha=2.7$ \\
\hline Variance & $\sigma_{\mathrm{dB}}^{2}=16 \mathrm{~dB}$ \\
\hline Carrier Frequency & $916 \mathrm{MHz}$ \\
\hline Antenna & omni \\
\hline Threshold (Sensitivity) & $\gamma=-118 \mathrm{~dB}$ \\
\hline \multicolumn{2}{|c|}{ Other Parameters } \\
\hline Reporting Frequency & $T_{r}=[0.1,1000] \mathrm{pps}^{1}$ \\
\hline Interface Queue Size & 50 packets \\
\hline UDP Packet Size & 100 bytes \\
\hline Detection Interval $\tau$ & $30 \mathrm{~s}$ \\
\hline
\end{tabular}

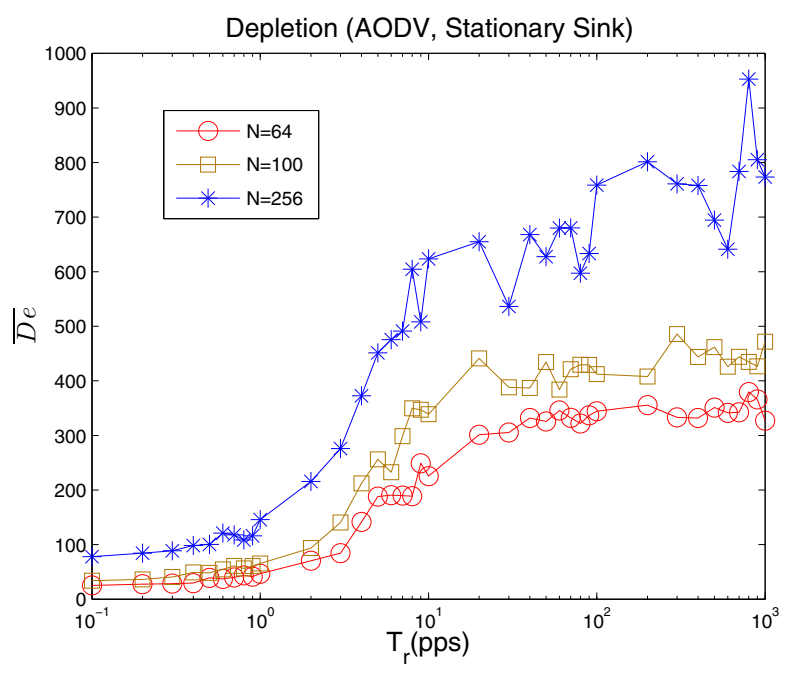

Figure 6. Depletion for stationary sink.

exploiting such links is possible but the performance gains are quite low. Thus, the routing protocol spends most of the time in the searching of a bi-directional path. Thus, given a fixed detection interval, $N_{r}$ can be much lower than its value in the case of the lattice topology, where the discovered paths do not change over time ${ }^{6}$. This fact may not affect the performance of the WSN, because it depends on the requirements of the application. For high values of $N$, the augmented interference level and the path instability seem to be predominant [14].

\section{Conclusions}

In this paper, we presented our simulation results of WSN for stationary and mobile sink considering two different topologies, lattice topology and random topology. We used consumed energy metric to measure the performance. From the simulation results, we conclude as follows:

${ }^{6}$ This is true if we do not count the reliability of nodes, i.e. the probability of failure of sensor nodes. 


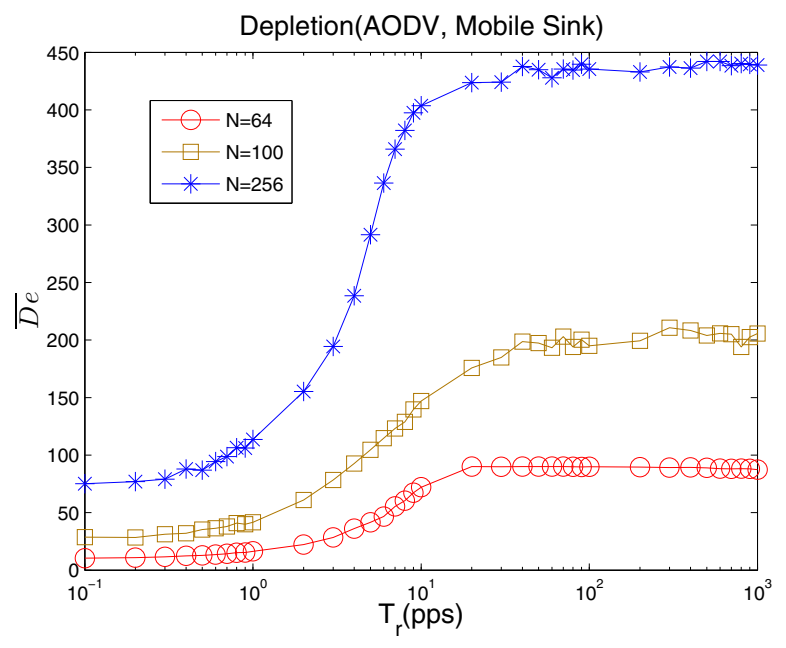

Figure 7. Depletion for mobile sink.

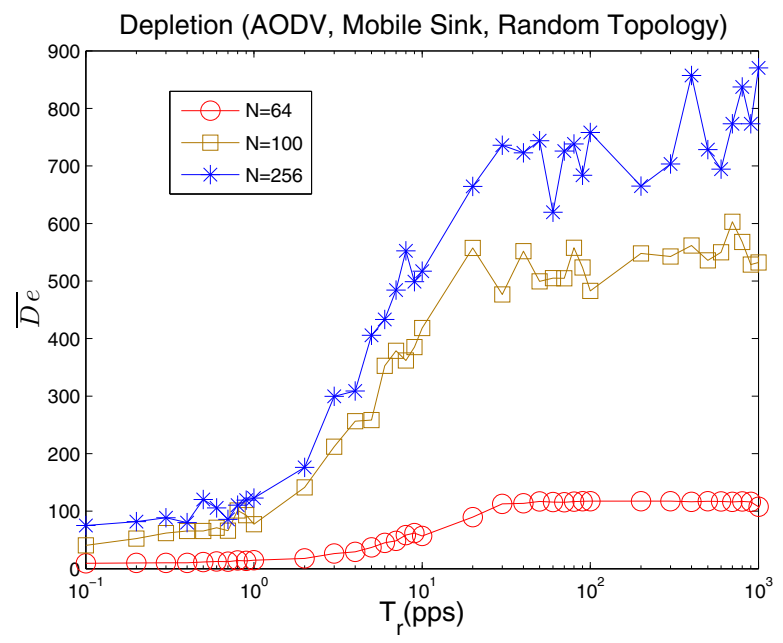

Figure 8. Depletion for mobile sink with random topology.

- In case of mobile sink, the consumed energy is better than stationary sink (about half of stationary sink).

- In case of mobile sink, the consumed energy of lattice topology is better than random topology.

In the future, we would like to carry out more extensive simulations for multi-mobile sinks. We also would like to consider the case of other routing protocols. Furthermore, we plan to consider the pathloss and routing efficiency metrics.

\section{ACKNOWLEDGMENT}

The authors would like to thank International Communications Foundation (ICF) of Japan and Japanese Society for the Promotion of Science (JSPS) for supporting this work.

\section{REFERENCES}

[1] S. Giordano and C. Rosenberg, "Topics in Ad Hoc and Sensor Networks", IEEE Communication Magazine, Vol. 44, No. 4, pp. 97-97, 2006.

[2] J. N. Al-Karaki and A. E. Kamal, "Routing Techniques in Wireless Sensor Networks: A Survey", IEEE Wireless Communication, Vol. 11, No. 6, pp. 6-28, December 2004.

[3] G. W.-Allen, K. Lorincz, O. Marcillo, J. Johnson, M. Ruiz, J. Lees, "Deploying a Wireless Sensor Network on an Active Volcano", IEEE Internet Computing, Vol. 10, No. 2, pp. 18-25, March, 2006.

[4] O. Younis, S. Fahmy, "HEED: A Hybrid, Energy-efficient, Distributed Clustering Approach for Ad-hoc Sensor Networks", IEEE Transactions on Mobile Computing, Vol. 3, No. 4, pp. 366-379, 2004.

[5] T. Yang, L. Barolli, G. De Marco, M. Ikeda, "Performance Evaluation of a Wireless Sensor Network for Mobile and Stationary Event Cases Considering Routing Efficiency and Goodput Metrics", Journal of Scalable Computing: Practice and Experience(SCPE), Vol. 10, No. 1, pp. 99-109, 2009.

[6] C. Perkins, "Ad Hoc Networks", Addison-Wesley, 2001.

[7] I. Donward, "NRL's Sensor Network Extension to NS-2", http://pf.itd.nrl.navy.mil/analysisnrlsensorsim/, 2004.

[8] Crossbow technology, inc. http://www.xbow.com/.

[9] T.S. Rappaport, "Wireless Communications", Prentice Hall PTR, 2001.

[10] W. Ye, J. Heidemann, D. Estrin, "Medium Access Control with Coordinated Adaptive Sleeping for Wireless Sensor Networks", IEEE/ACM Transaction Networking, Vol. 12, No. 3, pp. 493-506, 2004.

[11] Özgür B. Akan and I. F. Akyildiz "Event-to-Sink Reliable Transport in Wireless Sensor Networks", IEEE/ACM Transactions on Networking, Vol. 13, No. 5, pp. 1003-1016, 2005.

[12] G. Zhou, T. He, S. Krishnamurthy, J. A. Stankovic, "Models and Solutions for Radio Irregularity in Wireless Sensor Networks", ACM Transaction on Sensors Network, Vol. 2, No. 2, pp. 221-262, 2006.

[13] D. X. Wei. Speeding up ns-2 scheduler. http://netlab.caltech.edu/ weixl/technical/ns2patch, September 2005. California Institute of Technology.

[14] V.C. Gungor, M.C. Vuran, O.B. Akan, "On the Cross-layer Interactions Between Congestion and Contention in Wireless Sensor and Actor Networks", Ad Hoc Networks Journal (Elsevier), Vol. 5, No. 6, pp. 897-909, August 2007. 